pp. $78-84$

\title{
Desarrollo de un sistema fotovoltaico de generación eléctrica acoplado a un mecanismo de rotación automático (resultados preliminares)
}

\section{Ángel Arcia Gil ${ }^{*}$, Augusto Schlager ${ }^{2}$, Mateo Ramírez ${ }^{3}$}

${ }^{1}$ Profesor de Ingeniería Electrónica, Investigador, Universidad Católica Santa María, ${ }^{2}$ Egresado de Ingeniería Electrónica, Universidad Católica Santa María, ${ }^{3}$ Egresado de Ingeniería Electrónica, Investigador, Universidad Católica Santa María

*Autor para correspondencia. Email: aarciag@usma.com.pa

Recibido: 23 de julio de 2019

Aceptado: 9 de agosto de 2019

\section{Resumen}

La investigación en las áreas de energías renovables, específicamente de uso de energía solar fotovoltaica, es un área de fuerte desarrollo en la actualidad, debido a la necesidad de generar sistemas energéticos que permitan reducir el impacto de carbono que la actividad humana tiene sobre los ecosistemas. En este sentido, se desarrolló un prototipo de sistema fotovoltaico autónomo de seguimiento del sol, de poco procesamiento y bajo costo, buscando aumentar la capacidad de generación eléctrica de los paneles solares y hacer estudios comparativos de producción y análisis de factibilidad económica vs sistemas fijos. Adicionalmente, el proyecto permite a los estudiantes, profesores e investigadores involucrados, aprender conceptos complejos de manera práctica, diferenciando a la Universidad Católica Santa María La Antigua (USMA) como ente generador de innovación tecnológica, aportando resultados y diseños innovadores en el mercado de generación eléctrica solar de bajo consumo.

Palabras Clave: Sistema de Generación Solar, Sistema Fotovoltaico, Sistema de Rastreo Solar, Paneles Solares, Eficiencia Energética, Análisis de Sombra.

\section{Abstract \\ Research in areas such as renewable energies, specifically for the use of photovoltaic solar energy, is an area of strong development today, due to the need to create energy systems that reduce the impact of anthropogenic carbon emissions have on ecosystems. In this sense, a prototype of an autonomous photovoltaic system for tracking the Sun was developed, with little processing and low cost, seeking to increase the capacity of electricity generation of solar panels, as well as to make comparative studies of production and analysis of economic feasibility vs. fixed systems. Additionally, the project has}


allowed students, professors and researchers to learn complex concepts in a practical way, differentiating USMA as a generator of technological innovation and providing innovative results and designs to the low-power solar power generation market.

Key words: Solar Power Generation System, Solar Tracking System, Solar Panels, Energy Efficiency, Shadow Analysis

\section{Introducción}

Hoy en día existen diferentes fuentes de energía, las cuales se pueden catalogar en dos grandes grupos: convencionales y no convencionales. Las convencionales se refieren mayormente a los combustibles fósiles, mientras que las no convencionales pertenecen a las energías renovables, ej. energía solar, eólica, undimotriz, entre otras. Una de las tecnologías renovables que está a la vanguardia en cuanto a sistemas de generación eléctrica son los paneles fotovoltaicos. Estos paneles se componen de celdas fotovoltaicas hechas de silicio las cuales producen energía en forma eléctrica mediante la incidencia de la luz del Sol sobre las celdas [1]. El panel fotovoltaico obtiene su eficiencia máxima cuando los rayos del Sol inciden perpendicularmente en la superficie de las celdas fotovoltaicas, sin embargo, los rayos solares no inciden siempre de forma perpendicular en un plano fijo debido a la rotación de la Tierra, afectando la captación de rayos solares y conversión a energía eléctrica.

Se han realizados diferentes estudios [2] donde se han desarrollado sistemas para la rotación del panel fotovoltaico, que abarcan la rotación polar del panel (sistemas de un eje) y también sistemas que incluyen rotación polar y movimiento por elevación-azimut (sistema de dos ejes). En otras palabras, los sistemas de un eje siguen el sol de este a oeste, mientras que el sistema de dos ejes también sigue la altitud solar. Es importante destacar que los estudios han demostrado que los sistemas de seguimiento solar para los paneles fotovoltaicos logran percibir aproximadamente $30 \%$ más de energía que un panel estático [2]

Para crear un sistema de seguimiento solar eficiente, se necesitan cuatro componentes: sensores, software/mecanismo de seguimiento, motor y paneles fotovoltaicos. Algunos sistemas de seguimiento de posición solar utilizan sensores que permiten que el sistema de rotación pueda ajustarse de tal manera que la incidencia solar sea perpendicular a la superficie del módulo fotovoltaico. El sensor más utilizado es el sensor piranómetro, que mide con precisión la incidencia que existe sobre los rayos del sol en la tierra y la densidad del flujo de radiación solar en un campo de 180 grados. Debido al costo de este tipo de sensores, se utiliza en granjas solares donde la relación del costo-beneficio con respecto a un proyecto macro puede hacer que el costo del sensor sea muy bajo en relación al proyecto. Estos sensores tienen costos iniciales alrededor de los \$500 [3].

Otro tipo de sensores son los LDR (Light Dependent Resistor o fotorresistencia). Se han realizado diferentes estudios utilizando arreglos de LDR para detectar el cambio de posición del Sol [4] al igual que fotodiodos [5].

Se han realizado estudios donde se concluyen que la diferencia de grados entre el panel con respecto a la posición del Sol influye en la capacidad máxima de producción eléctrica del panel fotovoltaico. Dichos estudios mencionan que una desviación de 10 grados de la perpendicular proporciona medidas de aproximadamente 98,5\% de voltaje máximo, mientras que una desviación de 1 grado de la perpendicular genera aproximadamente $99,98 \%$ de voltaje máximo [6]. 
Otro mecanismo de seguimiento solar es el que utiliza algoritmos astronómicos de alta precisión para calcular el ángulo del sol, combinado con un microcontrolador de alto rendimiento, haciendo el sistema preciso y confiable, sin interferencia en días de lluvia, usando sensor de inclinación, retroalimentación lazo cerrado en tiempo real de seguimiento de ángulo y seguimiento automático sin intervención humana. El método de control es de cálculo preciso de Control de Tiempo. Con una precisión del seguidor es de hasta 0.5 Grados. [7].

En condiciones de falta de iluminación en días nublados o lluviosos, el seguidor puede calcular la posición real del sol de manera precisa y de esta manera, hacer el seguimiento de acuerdo con la longitud, latitud y tiempo usando algoritmos de alta precisión de ubicación del Sol.

Cuando el clima va de nublado a soleado, seguidor puede recoger toda la energía solar inmediatamente, realizando el seguimiento del Sol de manera más eficiente

En la USMA, ya existe un precedente de estudio de Producción de Energía con sistema Fotovoltaico a través del proyecto F.06-0019-A "Diseño y Construcción de Sistema Fotovoltaico para suministro de energía eléctrica”, desarrollado en 2010 por estudiantes de la Escuela de Electrónica con fondos de la Secretaría Nacional de Ciencias y Tecnología (SENACYT) [8].

El sistema implementado en 2010 contaba de una base con mecanismo de rotación Manual a $30^{\circ}, 90^{\circ}$, $120^{\circ}$. Se concluyó que un mecanismo de rotación ofrece mayor producción que un mecanismo fijo instalado en ángulo óptimo $\left(10^{\circ}-15^{\circ}\right.$ de inclinación SUR)
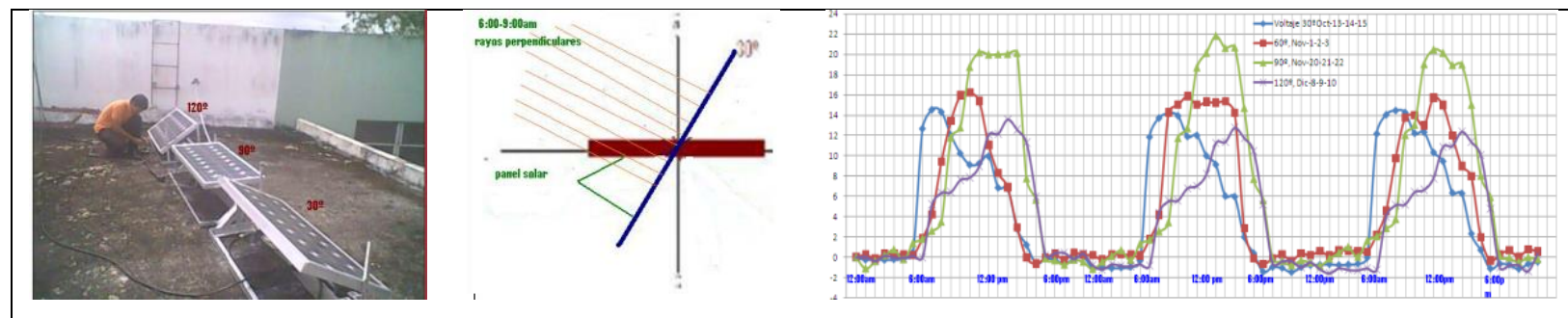

Fig. 1: Sistema de Rotación Manual implementado en Escuela de Electrónica, 2010.

A pesar de que el sistema de rotación manual es capaz de arrojar datos importantes para el estudio comparativo vs un sistema fijo, se hace necesario ajustar manualmente el ángulo cada 3 horas por lo que se desea implementar un mecanismo automático para la rotación y seguimiento.

Se analizaron 3 tipos/opciones de Arreglos para la estructura de rotación/seguimiento (fig.2a): Aseguimiento/rotación de 1 eje horizontal inclinado, B- seguimiento/rotación de 1 eje horizontal sin inclinación, C- seguimiento/rotación de 1 eje vertical sin inclinación

Se procedió a seleccionar el Arreglo A: Seguimiento/Rotación de 1 eje horizontal inclinado, debido a que éste da una producción similar a un sistema de rotación de 2 ejes en mayor rango de horas diarias (8:00 - 18:00 teórico, fig. 2b), es más simple de implementar y menos costos comparados con los arreglos $\mathrm{B}$ y C. La inclinación del sistema se estableció a $11^{\circ}$ hacia el SUR basados en los resultados del proyecto con rotación manual previamente implementado [8]. 


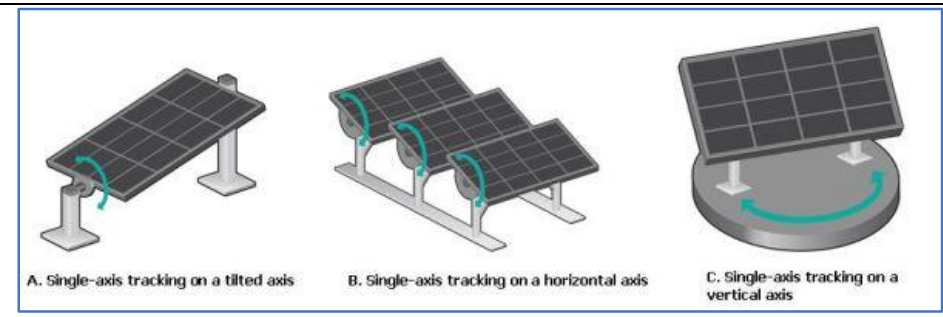

Fig. $2 a$ - Tipos de Layout para Estructura de Rotación

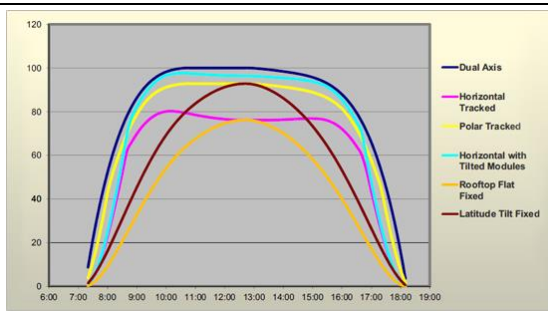

Fig. 2b - Comparativo de Producción Teórica de diferentes Layout

Se procedió a realizar diseño y fabricación de sistema de $1.6 \mathrm{~m}$ x $2 \mathrm{~m}$, inclinación $11^{\circ}$ al SUR, y se ensambló Prototipo en Laboratorio de Electrónica
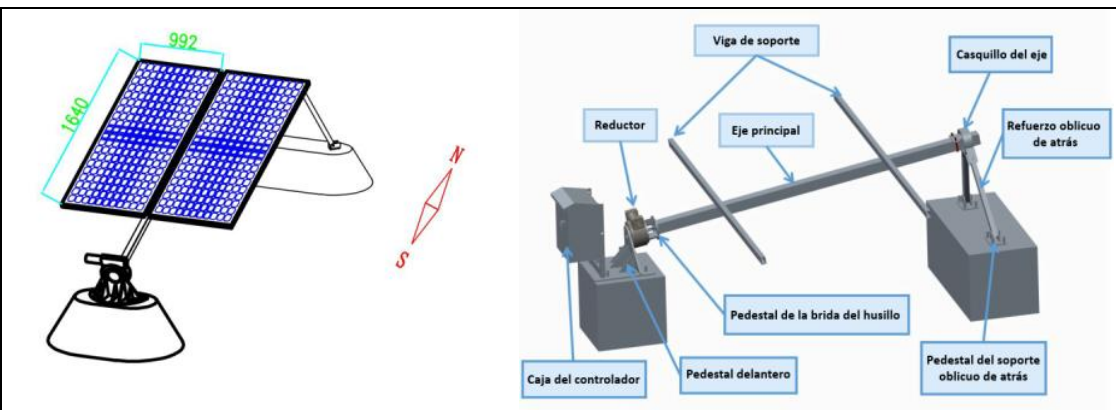

Fig. 3 a - Componentes del sistema de Rotación 1 Eje Horizontal Inclinado

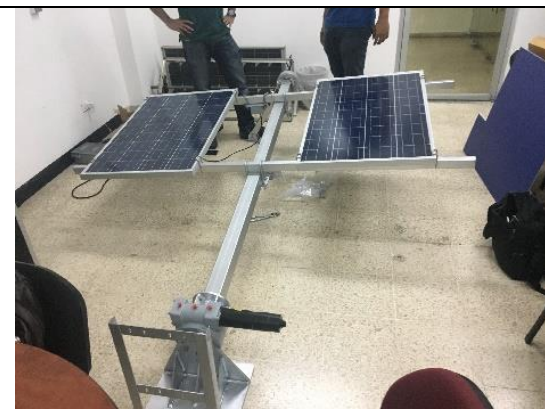

Fig. $3 b$ - Prototipo Ensamblado en Laboratorio

Se realizaron estudios iniciales de sombra y de producción energética para diversos sitios seleccionados dentro del campus USMA, así como análisis de Costos de Implementación vs Producción.

El primer sitio que se planteó para la realización del estudio fue el Puente entre Edificios D y E, tomando como referencia el sistema Anterior instalado (Lat.: 9.02939, Lon: -79.520915, Ele: 58 m)
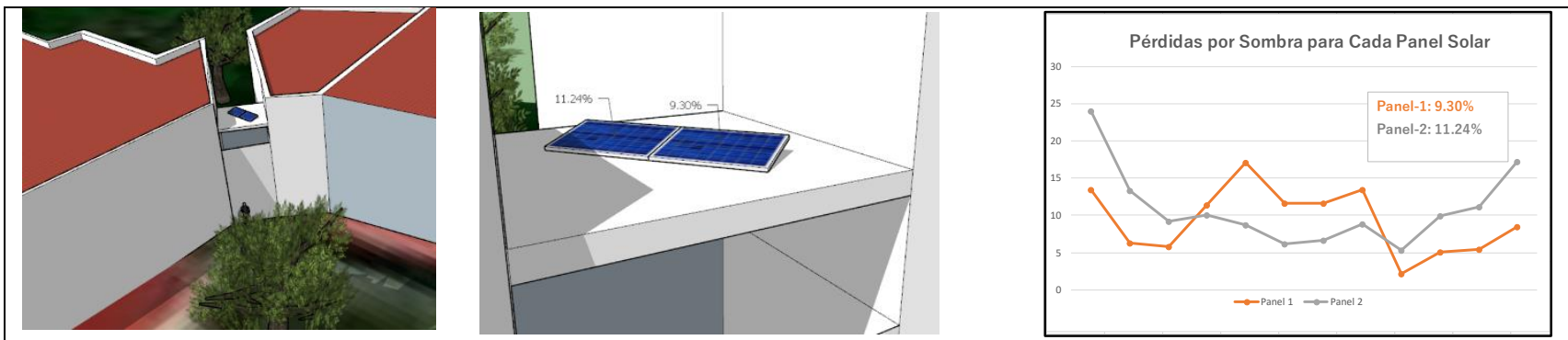

Fig. 4 - Análisis de Sombra y Producción en Puente entre Edificios D y E de la USMA

Tras varias réplicas de las mediciones y simulaciones con el Software Shadow Analysis for SketchUp se concluyó que el porcentaje de pérdidas era de aproximadamente un 19.32\%, por la interferencia del polvo, cableado, temperatura y conexiones y un porcentaje adicional de pérdida de $11.08 \%$ por sombra. 


\begin{tabular}{|c|c|c|c|c|c|c|c|c|c|c|c|c|c|c|c|c|c|c|c|c|c|c|}
\hline \multicolumn{10}{|c|}{$\mathrm{E}_{\mathrm{m}}(\mathrm{kWh} / \mathrm{month})$} & \multicolumn{13}{|c|}{$\mathrm{H}_{\mathrm{d}}\left(\mathrm{kWh} / \mathrm{m}^{2} /\right.$ day $)$} \\
\hline Face & Feb & Mar Apr & May Jun & Jul & Aug & Sep & Oct & Nov & Dec & Face & Jan & Feb & Mar & Apr & : May & Jun & Jul & Aug & Sep & Oct & Nov & Dec \\
\hline 1 & 22.5321 .01 & 23.4019 .89 & 17.4216 .01 & 16.38 & 817.89 & 16.95 & 18.20 & 15.52 & 218.33 & 1 & 5.89 & 6.19 & 6.17 & 5.49 & 4.69 & 4.43 & 4.39 & 4.81 & 4.72 & 4.79 & 4.29 & 4.89 \\
\hline$\Sigma$ & 22.5321 .01 & 23.4019 .89 & 17.4216 .01 & 16.38 & 817.89 & 16.95 & 518.20 & 15.52 & 218.33 & Mean & 5.89 & 96.19 & 6.17 & 5.49 & 4.69 & 4.43 & 4.39 & 4.81 & 4.72 & 2.79 & 4.29 & 4.89 \\
\hline
\end{tabular}

\begin{tabular}{|llllllllllll|l}
\hline \multicolumn{1}{l}{$\mathrm{H}_{\mathrm{m}}\left(\mathrm{kWh} / \mathrm{m}^{2} / \mathrm{month}\right)$} \\
Face
\end{tabular}

\begin{tabular}{|lllllll|}
\hline Groups global results & & & & \\
Solar panels & $\mathbf{N}^{\circ} \mathbf{P}$. & $\begin{array}{l}\text { P. power } \\
\text { (W) }\end{array}$ & $\begin{array}{l}\text { DC r. } \\
(\mathbf{k W p})\end{array}$ & $\begin{array}{l}\text { Energy Yield } \\
(\mathbf{k W h})\end{array}$ & $\begin{array}{l}\text { (kWh/kWp) } \\
(\mathbf{( \% )})\end{array}$ \\
Renesola:RS-SL-100TU-18P 2 & 100.00 & 0.20 & 223.55 & 1117.75 & 11.08 \\
\hline
\end{tabular}

Se concluyó que un porcentaje de pérdidas por sombra de $11.08 \%$ no permite hacer un estudio eficiente, en donde se pueda realizar la comparación entre el sistema fotovoltaico fijo y rotatorio, sin la acción del factor sombra. Por lo anterior, se sugirió realizar el estudio en un espacio libre de sombras.

La segunda ubicación analizada para la instalación del sistema autónomo de electrogeneración fotovoltaica fue la plaza Frontal de la Universidad en el estacionamiento de Profesores (Lat.: 9.029395, Lon: -79.520915 Ele: $58 \mathrm{~m})$.
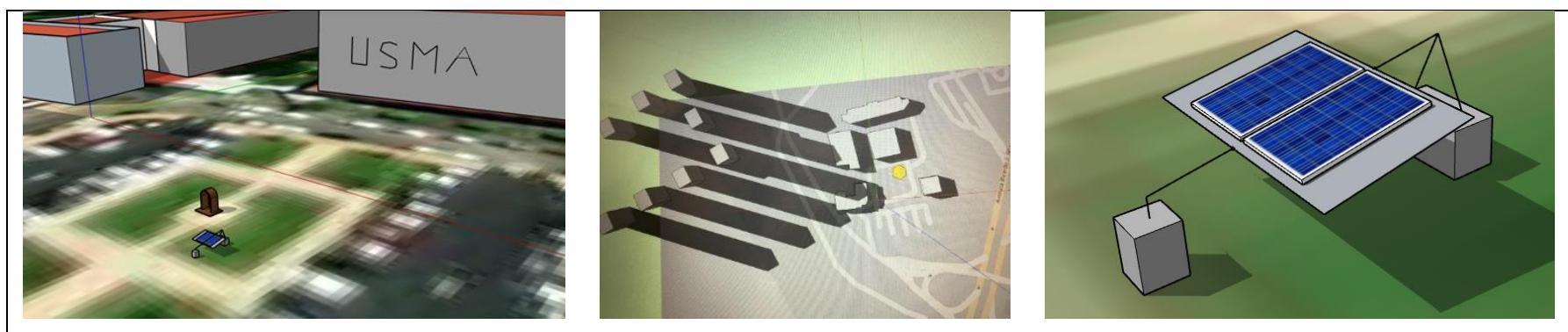

Fig. 5 - Análisis de Sombra y Producción en plaza de estacionamientos de profesores USMA

De los experimentos conducidos en esta locación, se concluyó que hubo un porcentaje promedio de pérdidas de alrededor de $21.66 \%$ por efectos del polvo, cableado, temperatura y conexiones; y un porcentaje de pérdida de $0.32 \%$ por sombra.

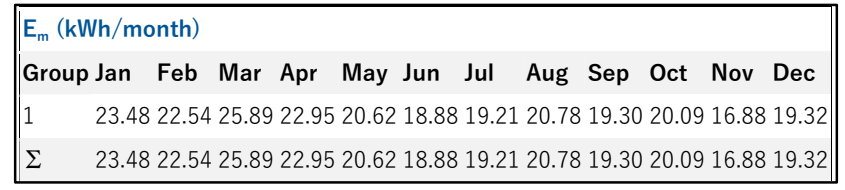

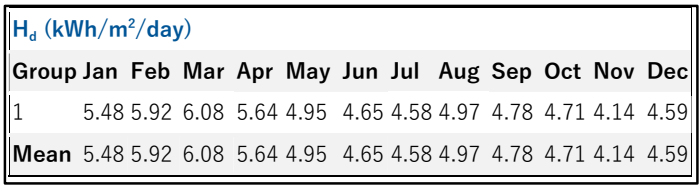




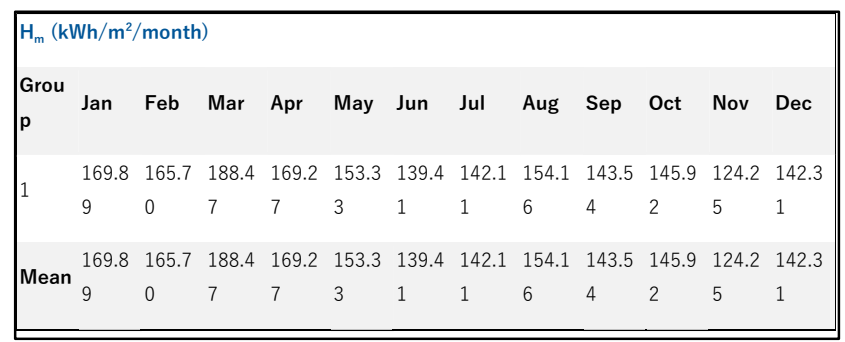

\begin{tabular}{|c|c|c|c|c|}
\hline \multicolumn{5}{|c|}{ Yearly average } \\
\hline \multirow{2}{*}{ Group } & Edy & Emy & Hdy & Hmy \\
\hline & (kWh/day & (kWh/month) & $\left(\mathrm{kWh} / \mathrm{m}^{2} /\right.$ day $)$ & $\left(\mathrm{kWh} / \mathrm{m}^{2} / \mathrm{month}\right)$ \\
\hline 1 & 0.68 & 20.83 & 5.04 & 153.34 \\
\hline$\Sigma$ or M & 0.68 & 20.83 & 5.04 & 153.34 \\
\hline
\end{tabular}

\begin{tabular}{|c|c|c|c|c|c|}
\hline \multicolumn{6}{|l|}{ Groups global results } \\
\hline Solar panels & $\begin{array}{l}\text { P. powe } \\
N^{\circ} P . \\
(W p)\end{array}$ & $\begin{array}{l}\text { DC r. } \\
(\mathrm{kWp})\end{array}$ & $\begin{array}{l}\text { Energy } \\
\text { (kWh) }\end{array}$ & $\begin{array}{l}\text { Yield } \\
(\mathrm{kWh} / \mathrm{kWp})\end{array}$ & $\begin{array}{l}\text { Shading L. } \\
\text { (\%) }\end{array}$ \\
\hline SunTracking_USMA 1 & 200.00 & 0.20 & 249.93 & 1249.65 & 0.32 \\
\hline
\end{tabular}

Se realizaron Análisis de Sombra a diferentes horas del día, (6am-6pm) y no se observaron mayores pérdidas por sombra (datos no mostrados, en proceso).

Se pudo determinar que el sistema rotatorio lograría una producción energética anual de 300.31 $\mathrm{kWh} /$ año. Si comparamos los resultados de este sistema rotatorio con un sistema fijo estándar, que logra una producción energética anual promedio de alrededor de $249.96 \mathrm{kWh} /$ año, se puede determinar que hubo un $\mathbf{1 6 . 7 7 \%}$ de mayor producción energética utilizando el sistema rotatorio, bajo las mismas condiciones (ubicación geográfica, inclinación, azimuth, modelo y cantidad de paneles solares).

Para el análisis económico se utilizaron 2 cotizaciones realizadas por la empresa Saiaz Panamá, para instalación del sistema de Seguimiento en el sitio-2 ya que de las pruebas y simulaciones de pérdidas por conexiones y por sombra resultaron más bajas que el sitio-1. Se analizaron 2 opciones: Opción-1: Instalación Tipo Estacionamiento y Opción-2: Instalación en Piso.

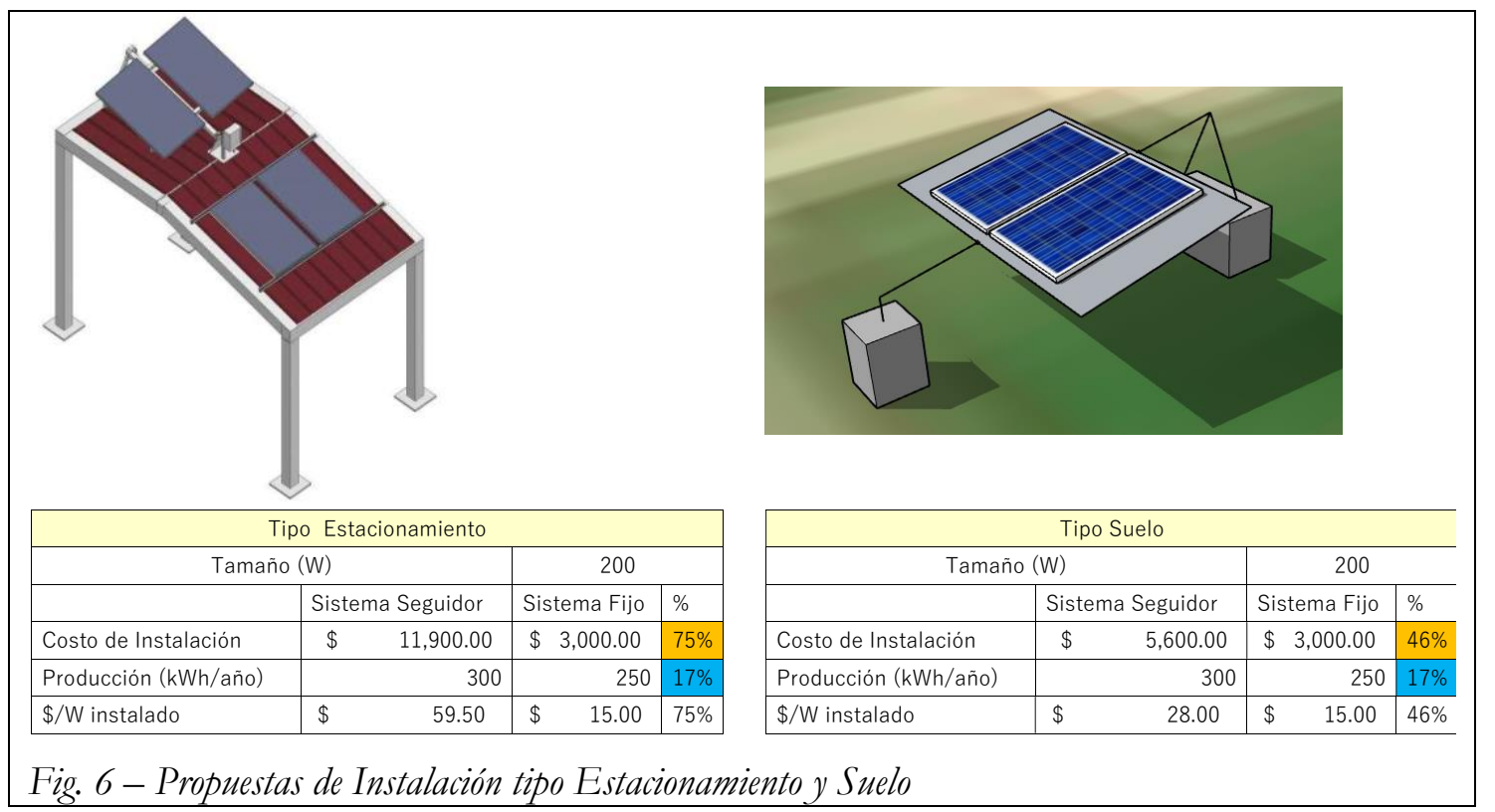


El sistema Seguidor tipo Estacionamiento produjo un 17\% más que su similar Sistema Fijo, sin embargo, requiere una inversión $75 \%$ mayor, lo que hace poco factible.

El sistema Seguidor tipo Suelo reduce el costo de instalación, sin embargo, el aumento en Producción $(17 \%)$ no es significativo en base al aumento del costo de Inversión (46\%).

Estos resultados preliminares indican la necesidad de buscar alternativas para hacer el sistema rotatorio, un sistema factible en la región de Panamá, con base a dos aspectos: 1. La mejora del diseño y la selección de ubicación para el emplazamiento del sistema, buscando un aumento de producción energética y 2. La mejora de los diseños específicamente para la reducción de costos.

\section{Bibliografía}

[1] IEEE Standard Definitions of Terms For Solar Cells," in IEEE Std No.307, vol., no., pp.1-4, 11 Nov. 1969

doi: 10.1109/IEEESTD.1969.120580

[2] T. Tudorache, C.D. Oancea, L. Kreindler, Performance Evaluation of a Solar Tracking PV Panel, U.P.B. Sci. Bull, Series C, Vol. 74, Iss. 1, 2012.

[3] A. Lay-Ekuakille, G. Vendramin, A. Fedele, L. Vasanelli, A. Trotta, PV Maximum Power Point Tracking Through Pyranometric Sensor: Modelling and Characterization, International Journal on Smart Sensing and Intelligent Systems, Vol. 1, No. 3, September 2008.

[4] S. Gagliano, N. Savalli, G. Tina and N. Pitrone, Two-axis Sun Tracking System: Design and Simulation, Eurosun 2006, Glasgow, June 2006.

[5] S. Gagliano, D. Neri, N. Pitrone, N. Savalli, G. Tina, Design of a Low-cost Solar Radiation Sensing Transducer for Photovoltaic Systems Planning and Monitoring. WSEAS TRANSACTIONS on ENVIRONMENT and DEVELOPMENT Issue 2, Volume 5, February 2009

[6] G. Oral, O. N. Uçan, Solar Tracking Systems and A Two-Axis Active Prototype with Stepwise Movement, International Journal of Electronics, Mechanical and Mechatronics Engineering, Vol. 3, No. 4.

[7] http://en.xinpengsolar.com/

[8] https://telecoss.com/projects/2010-usma-photovoltaic-system-implementation/ 